GLASNIK MATEMATIČKI

Vol. 41(61)(2006), $177-186$

\title{
LARGE TIME BEHAVIOR OF DIRICHLET HEAT KERNELS ON UNBOUNDED DOMAINS ABOVE THE GRAPH OF A BOUNDED LIPSCHITZ FUNCTION
}

\author{
KitTiPat Wong \\ Chulalongkorn University, Thailand
}

\begin{abstract}
Let $D \subseteq \mathbb{R}^{d}, d \geq 2$ be the unbounded domain above the graph of a bounded Lipschitz function. We study the asymptotic behavior of the transition density $p^{D}(t, x, y)$ of killed Brownian motions in $D$ and show that $\lim _{t \rightarrow \infty} t^{\frac{d+2}{2}} p^{D}(t, x, y)=C_{1} u(x) u(y)$, where $u$ is a minimal harmonic function corresponding to the Martin point at infinity and $C_{1}$ is a positive constant.
\end{abstract}

\section{IntRoduction}

This article is concerned with the large time asymptotic behavior of the Dirichlet heat kernel $p^{D}(t, x, y)$ on the unbounded domain $D$ above the graph of a bounded Lipschitz function $f$. Here and in the sequel, by a domain in $\mathbb{R}^{d}, d \geq 2$, we mean an open connected subset of $\mathbb{R}^{d}$. The Dirichlet heat kernel is the transition density of the Brownian motion killed upon leaving the domain $D$. This work was inspired by the results of Pierre Collet, Servet Martínez and Jaime San Martín [2]. Those authors obtained the asymptotic behavior of the Dirichlet heat kernel on an exterior domain, i.e., an unbounded domain which is the complement of a compact nonpolar subset of $\mathbb{R}^{d}$. More specifically, they proved that for $x, y$ in the plannar exterior domain $D$,

$$
\lim _{t \uparrow \infty} t(\log t)^{2} p^{D}(t, x, y)=\frac{2}{\pi} u_{1}(x) u_{1}(y),
$$

where $u_{1}(x)=\pi \lim _{|y| \rightarrow \infty} G^{D}(x, y)$ and $G^{D}(x, y)=\int_{0}^{\infty} p^{D}(t, x, y) d t$. Those authors also obtained the result in higher dimensions, namely, for $d \geq 3$, and

2000 Mathematics Subject Classification. 60J65, 35J05.

Key words and phrases. Dirichlet heat kernels, asymptotic behavior, Brownian motions. 
$x, y \in D$,

$$
\lim _{t \uparrow \infty} t^{\frac{d}{2}} p^{D}(t, x, y)=(2 \pi)^{-\frac{d}{2}} u_{2}(x) u_{2}(y),
$$

where $u_{2}(x)=\lim _{t \uparrow \infty} P_{x}\left(\tau_{D}>t\right)$ and $\tau_{D}$ is the first exit time of the exterior domain $D$. We adapt the techniques used by Collet, Martínez and San Martín to obtain the asymptotic behavior of the Dirichlet heat kernel in the case of the unbounded domain $D$ above the graph of a bounded Lipschitz function. Our main result on the asymptotic behavior of the Dirichlet heat kernel $p^{D}(t, x, y)$ on the unbounded domain above the graph of a bounded Lipschitz function is the following (see Theorem 2.5 for more details). Let $D \subseteq \mathbb{R}^{d}$ be the domain above the graph of a bounded Lipschitz function $f$. Then, for any $x, y \in D$, we have

$$
\lim _{t \uparrow \infty} t^{\frac{d+2}{2}} p^{D}(t, x, y)=H u(x) u(y),
$$

for some positive constant $H$ and positive harmonic function $u$ vanishing on the boundary $\partial D$ of $D$.

\section{MAin RESUlts}

In this section, we are going to establish our main results. We fix a bounded real-valued Lipschitz function $f: \mathbb{R}^{d-1} \rightarrow \mathbb{R}$. Recall that a Lipschitz function $f$ on $\mathbb{R}^{d-1}$ means that there exists a positive constant $C$ such that

$$
\left|f\left(\tilde{x}_{1}\right)-f\left(\tilde{x}_{2}\right)\right| \leq C\left|\tilde{x}_{1}-\tilde{x}_{2}\right|,
$$

for all $\tilde{x}_{1}, \tilde{x}_{2} \in \mathbb{R}^{d-1}$. We also fix a domain $D \subseteq \mathbb{R}^{d}$ as follows:

$$
D=\left\{x=\left(\tilde{x}, x_{d}\right) \in \mathbb{R}^{d-1} \times \mathbb{R}=\mathbb{R}^{d}: x_{d}>f(\tilde{x})\right\} .
$$

The unbounded domain $D$ above is called the domain above the graph of a bounded Lipschitz function. Since $f$ is bounded, there exist constants $a$ and $b$ such that

for each $\tilde{x} \in \mathbb{R}^{d-1}$. Define

$$
a \leq f(\tilde{x}) \leq b,
$$

$$
\begin{gathered}
\mathcal{H}=\{h: h \text { is nonnegative and harmonic in } D \text { and } \\
\left.\lim _{D \ni x \rightarrow y} h(x)=0, \text { for all } y \in \partial D\right\} .
\end{gathered}
$$

Notice that $\partial D=\left\{x=\left(\tilde{x}, x_{d}\right) \in \mathbb{R}^{d}: x_{d}=f(\tilde{x})\right\}$.

TheOREM 2.1. There is only one point corresponding to infinity on the Martin boundary of $D$ and this Martin point is minimal. In particular, this means that $\mathcal{H}$ defined above is one dimensional. 
Proof. Fix a point $z_{0}=\left(\tilde{z}_{0}, a-2\right) \in \mathbb{R}^{d}$ and consider the inversion with respect to the sphere $S_{1}\left(z_{0}\right):=\left\{x \in \mathbb{R}^{d}:\left|x-z_{0}\right|=1\right\}$. The image $D^{*}$ of $D$ under this inversion is a bounded Lipschitz domain with $z_{0}$ being the image of infinity. Notice that $z_{0}$ is on the boundary of $D^{*}$. By Theorem 1.5 on page 337 in [5], the Martin boundary, the minimal Martin boundary and the Euclidean boundary of $D^{*}$ coincide. This means that each boundary point $z$ of $D^{*}$ corresponds to a minimal harmonic function $v_{z}$. Define a function $u_{z}(x)=\left|x^{*}-z_{0}\right|^{d-2} v_{z}\left(x^{*}\right)$, for $x \in D$ and $x^{*}=z_{0}+\frac{1}{\left|x-z_{0}\right|^{2}}\left(x-z_{0}\right)$. Then, the Laplacian of $u_{z}$ vanishes on $D$ (see [1]) and therefore, $\mathcal{H}$ is one dimensional. Moreover, there is only one point corresponding to infinity on the Martin boundary of $D$ and this Martin point is minimal.

REMARK 2.2. From now on we will use $u$ to denote a positive harmonic function in $D$ corresponding to the Martin point at infinity.

We will obtain some property of the function $u$ after the following observation.

Let us recall the Green function $G_{H}$ for the half space $H:=\left\{x=\left(\tilde{x}, x_{d}\right) \in\right.$ $\left.\mathbb{R}^{d}: x_{d}>0\right\}$. For $x, y \in H$ with $x \neq y$,

$$
G_{H}(x, y)=k(x, y)-k\left(x, y^{\prime}\right)
$$

where

$$
k(x, y)= \begin{cases}\frac{1}{\pi} \ln \left(\frac{1}{|x-y|}\right) & \text { if } d=2 \\ |x-y|^{2-d} & \text { if } d \geq 3\end{cases}
$$

and $y^{\prime}=\left(y_{1}, y_{2}, \ldots, y_{d-1},-y_{d}\right)$ (see page 113 in $\left.[6]\right)$. Then, for $d=2$,

$$
G_{H}(x, y)=\frac{1}{\pi} \ln \left(\frac{\left|x-y^{\prime}\right|}{|x-y|}\right) .
$$

Notice that

$$
\begin{aligned}
\left|x-y^{\prime}\right|^{2} & =|x-y|^{2}+4 x_{d} y_{d} \\
& =|x-y|^{2}(1+\beta),
\end{aligned}
$$

where $\beta=\frac{4 x_{d} y_{d}}{|x-y|^{2}}>0$. Therefore,

$$
\begin{aligned}
G_{H}(x, y) & =\frac{1}{\pi} \ln \left(\frac{|x-y| \sqrt{1+\beta}}{|x-y|}\right) \\
& =\frac{1}{2 \pi} \ln \left(1+\frac{4 x_{d} y_{d}}{|x-y|^{2}}\right) .
\end{aligned}
$$

The above equation will be used in the proof of the next theorem. The notation $f_{1} \approx f_{2}$ means that there exists a positive constant $C$ such that $\frac{1}{C} f_{2} \leq f_{1} \leq C f_{2}$. 
TheOREM 2.3. For sufficiently large $x_{d}$, we have

$$
u\left(\tilde{x}, x_{d}\right) \approx x_{d} .
$$

Proof. Let $x_{0}=(\tilde{0}, b+100) \in D$ and $y_{n}=(\tilde{0}, b+n) \in D$. Then, Theorem 1.5 on page 337 in [5] implies that for any $x \in D$,

$$
\lim _{n \rightarrow \infty} \frac{G_{D}\left(x, y_{n}\right)}{G_{D}\left(x_{0}, y_{n}\right)}=u(x) \text {. }
$$

Define

$$
D_{a}=\left\{x=\left(\tilde{x}, x_{d}\right): x_{d}>a\right\}, \quad D_{b}=\left\{x=\left(\tilde{x}, x_{d}\right): x_{d}>b\right\} .
$$

Then, for any $x \in D_{b}$, we have

$$
G_{D_{b}}\left(x, y_{n}\right) \leq G_{D}\left(x, y_{n}\right) \leq G_{D_{a}}\left(x, y_{n}\right)
$$

Thus, for any $x \in D_{b}$,

$$
\liminf _{n \rightarrow \infty} \frac{G_{D_{b}}\left(x, y_{n}\right)}{G_{D_{a}}\left(x_{0}, y_{n}\right)} \leq u(x) \leq \limsup _{n \rightarrow \infty} \frac{G_{D_{a}}\left(x, y_{n}\right)}{G_{D_{b}}\left(x_{0}, y_{n}\right)} .
$$

From the explicit formulae for $G_{D_{a}}$ and $G_{D_{b}}$, it can be shown that for $d \geq 3$

$$
\begin{aligned}
& G_{D_{a}}(x, y) \approx|x-y|^{2-d} \min \left\{1, \frac{\left(x_{d}-a\right)\left(y_{d}-a\right)}{|x-y|^{2}}\right\}, \\
& G_{D_{b}}(x, y) \approx|x-y|^{2-d} \min \left\{1, \frac{\left(x_{d}-b\right)\left(y_{d}-b\right)}{|x-y|^{2}}\right\} .
\end{aligned}
$$

Thus, for any $x \in D_{b}$,

$$
u(x) \leq C \limsup _{n \rightarrow \infty} \frac{\frac{\left(x_{d}-a\right)\left(y_{d}^{(n)}-a\right)}{\left|x-y_{n}\right|^{2}} \wedge 1}{\frac{\left(x_{d}^{(0)}-b\right)\left(y_{d}^{(n)}-b\right)}{\left|x_{0}-y_{n}\right|^{2}} \wedge 1}=C \frac{x_{d}-a}{100}
$$

where $x_{d}^{(0)}$ and $y_{d}^{(n)}$ are the $d$-th component of $x_{0}$ and $y_{n}$, respectively. Also,

$$
\begin{aligned}
u(x) & \geq C \liminf _{n \rightarrow \infty} \frac{\frac{\left(x_{d}-b\right)\left(y_{d}^{(n)}-b\right)}{\left|x-y_{n}\right|^{2}} \wedge 1}{\frac{\left(x_{d}^{(0)}-a\right)\left(y_{d}^{(n)}-a\right)}{\left|x_{0}-y_{n}\right|^{2}} \wedge 1} \\
& =C \frac{x_{d}-b}{x_{d}^{(0)}-a}=C \frac{x_{d}-b}{b+100-a} .
\end{aligned}
$$

For $d=2$, it follows from (2.3) that the Green functions $G_{D_{a}}$ and $G_{D_{b}}$ for $D_{a}$ and $D_{b}$ respectively are given by

$$
\begin{aligned}
G_{D_{a}}(x, y) & =\frac{1}{2 \pi} \ln \left(1+\frac{4\left(x_{d}-a\right)\left(y_{d}-a\right)}{|x-y|^{2}}\right) \text { and } \\
G_{D_{b}}(x, y) & =\frac{1}{2 \pi} \ln \left(1+\frac{4\left(x_{d}-b\right)\left(y_{d}-b\right)}{|x-y|^{2}}\right)
\end{aligned}
$$


Recall the fact that $\ln (1+x) \approx x$ as $x \rightarrow 0$. By (2.4),

$$
\begin{aligned}
u(x) & \leq \limsup _{n \rightarrow \infty} \frac{\ln \left(1+\frac{4\left(x_{d}-a\right)\left(y_{d}^{(n)}-a\right)}{\left|x-y_{n}\right|^{2}}\right)}{\ln \left(1+\frac{4\left(x_{d}^{(0)}-b\right)\left(y_{d}^{(n)}-b\right)}{\left|x_{0}-y_{n}\right|^{2}}\right)} \\
& =\limsup _{n \rightarrow \infty} \frac{\ln \left(1+\frac{4\left(x_{d}-a\right)(b+n-a)}{\left|x-y_{n}\right|^{2}}\right)}{\ln \left(1+\frac{4(100)(b+n-b)}{\left|x_{0}-y_{n}\right|^{2}}\right)} \leq C x_{d},
\end{aligned}
$$

for some positive constant $C$, since both $\frac{4\left(x_{d}-a\right)(b+n-a)}{\left|x-y_{n}\right|^{2}}$ and $\frac{4(100)(b+n-b)}{\left|x_{0}-y_{n}\right|^{2}}$ converge to 0 as $n \rightarrow \infty$.

Similarly,

$$
\begin{aligned}
u(x) & \geq \liminf _{n \rightarrow \infty} \frac{\ln \left(1+\frac{4\left(x_{d}-b\right)\left(y_{d}^{(n)}-b\right)}{\left|x-y_{n}\right|^{2}}\right)}{\ln \left(1+\frac{4\left(x_{d}^{(0)}-a\right)\left(y_{d}^{(0)}-a\right)}{\left|x_{0}-y_{n}\right|^{2}}\right)} \\
& =\liminf _{n \rightarrow \infty} \frac{\ln \left(1+\frac{4\left(x_{d}-b\right)(n)}{\left|x-y_{n}\right|^{2}}\right)}{\ln \left(1+\frac{4(b+100-a)(b+n-a)}{\left|x_{0}-y_{n}\right|^{2}}\right)}=C x_{d},
\end{aligned}
$$

for some positive constant $C$.

THEOREM 2.4. The function u defined above is invariant, i.e.,

$$
u(x)=\int_{D} p^{D}(t, x, y) u(y) d y
$$

for all $t>0$ and $x \in D$.

Proof. To show that $u$ is invariant, it suffices to show (see pages 728 and 669 in [3]) that for some $x \in D$,

$$
\lim _{t \uparrow \infty} \int_{D} p^{D}(t, x, y) u(y) d y>0
$$

In other words, the condition above means that the function $u$ is not purely excessive. By Theorem 2.3, take $M>b$ so large that

$$
u(y) \approx y_{d} \quad \text { for } y_{d}>M
$$


and put $x=(\tilde{0}, 1+M) \in D$. Then, we obtain

$$
\begin{aligned}
\int_{D} p^{D}(t, x, y) u(y) d y & \geq \int_{D_{b}} p^{D}(t, x, y) u(y) d y \\
& \geq \int_{D_{b}} p^{D_{b}}(t, x, y) u(y) d y \\
& \geq C \int_{\left\{y: y_{d}>M\right\}} p^{D_{M}}(t, x, y) y_{d} d y .
\end{aligned}
$$

By the explicit formula of

$$
p^{D_{M}}(t, x, y)=C\left(\frac{x_{d}-M}{\sqrt{t}} \wedge 1\right)\left(\frac{y_{d}-M}{\sqrt{t}} \wedge 1\right) t^{-\frac{d}{2}} \exp \left(-\frac{|x-y|^{2}}{2 t}\right)
$$

and for $t$ so large that $1-\frac{1}{\sqrt{t}}>\frac{1}{2}$ and $\frac{1}{\sqrt{t}} \wedge 1=\frac{1}{\sqrt{t}}$, we have

$$
\begin{aligned}
& \int_{\left\{y: y_{d}>M\right\}} p^{D_{M}}(t, x, y) y_{d} d y \\
& \geq C \int_{\left\{y: y_{d}>M\right\}}\left(\frac{1}{\sqrt{t}} \wedge 1\right)\left(\frac{y_{d}-M}{\sqrt{t}} \wedge 1\right) t^{-\frac{d}{2}} \exp \left(-\frac{|x-y|^{2}}{2 t}\right)\left(y_{d}-M\right) d y \\
& =C \frac{1}{\sqrt{t}} \int_{\left\{y: y_{d}>M\right\}}\left(\frac{y_{d}-M}{\sqrt{t}} \wedge 1\right)\left(y_{d}-M\right) t^{-\frac{d}{2}} \exp \left(-\frac{|x-y|^{2}}{2 t}\right) d y \\
& =C \frac{1}{\sqrt{t}} \int_{M}^{\infty}\left(\frac{y_{d}-M}{\sqrt{t}} \wedge 1\right)\left(y_{d}-M\right) t^{-\frac{1}{2}} \exp \left(-\frac{\left|y_{d}-M-1\right|^{2}}{2 t}\right) d y_{d} \\
& \geq C \frac{1}{t} \int_{M}^{M+\sqrt{t}}\left(y_{d}-M\right)^{2} t^{-\frac{1}{2}} \exp \left(-\frac{\left|y_{d}-M-1\right|^{2}}{2 t}\right) d y_{d} \\
& \geq C \frac{1}{t} \int_{M}^{M+\sqrt{t}}\left(y_{d}-M-1\right)^{2} t^{-\frac{1}{2}} \exp \left(-\frac{\left|y_{d}-M-1\right|^{2}}{2 t}\right) d y_{d} \\
& =C \frac{1}{t} \int_{-1}^{\sqrt{t}-1} v^{2} t^{-\frac{1}{2}} \exp \left(-\frac{v^{2}}{2 t}\right) d v \\
& =C \int_{-\frac{1}{\sqrt{t}}}^{1-\frac{1}{\sqrt{t}}} u^{2} \exp \left(-\frac{u^{2}}{2}\right) d u \\
& >C \int_{0}^{\frac{1}{2}} u^{2} \exp \left(-\frac{u^{2}}{2}\right) d u>0 .
\end{aligned}
$$

Let $d(x)$ be the Euclidean distance from $x$ to the boundary $\partial D$ of $D$ and recall that the function $u$ is the Martin kernel corresponding to the point at infinity and $x_{0}=(\tilde{0}, b+100) \in D$. Now, we are ready to establish the main result. 
Theorem 2.5. Let $D \subseteq \mathbb{R}^{d}, d \geq 2$, be the domain above the graph of $f$. For any $x, y \in D$, we have

$$
\lim _{t \uparrow \infty} t^{\frac{d+2}{2}} p^{D}(t, x, y)=C u(x) u(y),
$$

where $u$ is the function introduced in Remark 2.2 and $C$ is a positive constant. The convergence is uniform on compact subsets of $D \times D$.

Proof. Let $x, y \in D$ and fix $x_{0}=(\tilde{0}, b+100) \in D \cap D_{b}=D_{b}$. It is easy to see from the explicit formula of the Dirichlet heat kernel on the half space $D_{a}$ that $p^{D_{a}}\left(t, x_{0}, x_{0}\right) \geq p^{D_{a}}(t, x, y)$. Therefore,

$$
\frac{p^{D}(t, x, y)}{p^{D_{a}}\left(t, x_{0}, x_{0}\right)} \leq \frac{p^{D}(t, x, y)}{p^{D_{a}}(t, x, y)} \leq 1 .
$$

Let $K$ be a compact subset of $D$. Then, there exists $k \in \mathbb{N}$ such that $K \subseteq D_{k}$, where

$$
D_{k}=\left\{x \in D: d(x)>\frac{1}{k},|\tilde{x}| \leq k \text { and } x_{d} \leq b+100+k\right\} .
$$

Therefore, the parabolic Harnack inequality (see Theorem 1 in [4]) implies the existence of a positive constant $C$ such that for $x, y \in K$ and $t>t_{0}$, for some positive $t_{0}$,

$$
\begin{aligned}
p^{D}(t, x, y) & \geq C p^{D}\left(t-\varepsilon, x_{0}, y\right) \\
& \geq C p^{D}\left(t-\varepsilon, x_{0}, x_{0}\right) \\
& \geq C p^{D_{b}}\left(t-\varepsilon, x_{0}, x_{0}\right)
\end{aligned}
$$

for some $\varepsilon>0$. Notice that we can assume that $t_{0}>\varepsilon$.

So, we have as $t \uparrow \infty$,

$$
\begin{aligned}
\frac{p^{D}(t, x, y)}{p^{D_{a}}\left(t, x_{0}, x_{0}\right)} & \geq C \frac{p^{D_{b}}\left(t-\varepsilon, x_{0}, x_{0}\right)}{p^{D_{a}}\left(t, x_{0}, x_{0}\right)} \\
& \longrightarrow C \frac{\left(x_{d}^{(0)}-b\right)^{2}}{\left(x_{d}^{(0)}-a\right)^{2}}>0
\end{aligned}
$$

where $x_{d}^{(0)}$ is the $d$-th component of $x_{0}$. Thus, the family of functions $\left\{\frac{p^{D}(t, x, y)}{p^{D_{a}\left(t, x_{0}, x_{0}\right)}}: t>t_{0}\right\}$ is bounded on compact subsets of $D \times D$. Next, we claim that

$$
\sup _{t \geq t_{0},|s| \leq 2} \frac{p^{D_{a}}\left(t, x_{0}, x_{0}\right)}{p^{D_{a}}\left(t+s, x_{0}, x_{0}\right)}<\infty
$$


To see this, for sufficiently large $t>t_{0}$ and $|s| \leq 2$,

$$
\begin{aligned}
\frac{p^{D_{a}}\left(t, x_{0}, x_{0}\right)}{p^{D_{a}}\left(t+s, x_{0}, x_{0}\right)} & =\frac{\frac{2}{(2 \pi)^{d / 2}}(b+100-a)^{2} t^{-\frac{d+2}{2}}}{\frac{2}{(2 \pi)^{d / 2}}(b+100-a)^{2}(t+s)^{-\frac{d+2}{2}}} \\
& =\left(\frac{t+s}{t}\right)^{\frac{d+2}{2}}=\left(1+\frac{s}{t}\right)^{\frac{d+2}{2}}<\infty .
\end{aligned}
$$

Therefore, the family of functions $\left\{\frac{p^{D}(t, x, y)}{p^{D_{a}\left(t, x_{0}, x_{0}\right)}}: t>t_{0}\right\}$ is equicontinuous on compact subsets of $D \times D$ by Lemma 2.1 in [2]. Therefore, Arzela-Ascoli theorem implies that any sequence converging to infinity contains a subsequence $t_{n} \uparrow \infty$ such that

$$
\lim _{t_{n} \uparrow \infty} \frac{p^{D}\left(t_{n}, x, y\right)}{p^{D_{a}}\left(t_{n}, x_{0}, x_{0}\right)}=V(x, y)
$$

for some continuous function $V(\cdot, y)$, where the convergence is uniform on compact subsets of $D$. Note that $V(x, y) \geq C \frac{10^{4}}{(b+100-a)^{2}}>0$ and therefore $V(x, y)$ is nontrivial.

From the semigroup property, we get that for any $s>0$,

$$
\frac{p^{D}\left(t_{n}+s, x, y\right)}{p^{D_{a}}\left(t_{n}, x_{0}, x_{0}\right)}=\int_{D} \frac{p^{D}\left(t_{n}, x, \xi\right)}{p^{D_{a}}\left(t_{n}, x_{0}, x_{0}\right)} \cdot p^{D}(s, \xi, y) d \xi
$$

Recall from (2.5) that $\frac{p^{D}(t, x, y)}{p^{D_{a}\left(t, x_{0}, x_{0}\right)}} \leq 1$ for all $t>0 ; x, y \in D$ and $x_{0} \in D_{b}$. Using this inequality, the Gaussian bound for $p^{D}$ and the dominated convergence theorem, we obtain

$$
V(x, y)=\int_{D} V(x, \xi) p^{D}(s, \xi, y) d \xi .
$$

Therefore, $V(\cdot, y)$ is a nontrivial positive harmonic function vanishing at the boundary $\partial D$ of $D$. Since $\mathcal{H}$ is one dimensional by Theorem $2.1, V(\cdot, y)=$ $a(y) u(\cdot)$ for some function $a=a(y)$. By the symmetry of the problem, we have

$$
V(x, y)=C_{1} u(x) u(y)
$$

for some positive constant $C_{1}$, which may depend on the subsequence. So,

$$
\lim _{t_{n} \uparrow \infty} \frac{p^{D}\left(t_{n}, x, y\right)}{p^{D_{a}}\left(t_{n}, x_{0}, x_{0}\right)}=C_{1} u(x) u(y) .
$$

On the other hand, by the explicit formula of $p^{D_{a}}$, we obtain

$$
\lim _{t_{n} \uparrow \infty} \frac{p^{D}\left(t_{n}, x, y\right)}{p^{D_{a}}\left(t_{n}, x_{0}, x_{0}\right)}=\lim _{t_{n} \uparrow \infty} \frac{t_{n}^{\frac{d+2}{2}} p^{D}\left(t_{n}, x, y\right)}{\frac{2}{(2 \pi)^{d / 2}}(b+100-a)^{2}} .
$$


So,

$$
\lim _{t_{n} \uparrow \infty} \frac{t_{n}^{\frac{d+2}{2}} p^{D}\left(t_{n}, x, y\right)}{\frac{2}{(2 \pi)^{d / 2}}(b+100-a)^{2}}=C_{1} u(x) u(y) .
$$

Hence,

$$
\lim _{t_{n} \uparrow \infty} t_{n}^{\frac{d+2}{2}} p^{D}\left(t_{n}, x, y\right)=C u(x) u(y),
$$

where $C=\frac{2 C_{1}}{(2 \pi)^{d / 2}}(b+100-a)^{2}$. Notice that

$$
t_{n}^{\frac{d+2}{2}} p^{D_{b}}\left(t_{n}, y, y\right) \leq t_{n^{2}}^{\frac{d+2}{2}} p^{D}\left(t_{n}, y, y\right) \leq t_{n}^{\frac{d+2}{2}} p^{D_{a}}\left(t_{n}, y, y\right) .
$$

Taking $t_{n} \uparrow \infty$ and $y_{d}$ being sufficiently large give us

$$
\frac{2}{(2 \pi)^{d / 2}}\left(y_{d}-b\right)^{2} \leq C(u(y))^{2} \leq \frac{2}{(2 \pi)^{d / 2}}\left(y_{d}-a\right)^{2} .
$$

Since $u(y)$ behaves asymptotically as $y_{d}$ by Theorem 2.3 , we see that the limit of $t_{n}^{\frac{d+2}{2}} p^{D}\left(t_{n}, y, y\right)$ as $t \uparrow \infty$ does not depend on the subsequence $t_{n}$, and we conclude that

$$
\lim _{t \uparrow \infty} t^{\frac{d+2}{2}} p^{D}(t, x, y)=C u(x) u(y) .
$$

The convergence is uniform on compact subsets of $D \times D$.

\section{ACKNowledgements.}

I would like to thank Professor Renming Song for the guidance and discussion of this paper. Also, many thanks go to the referees on the comments of this paper.

\section{REFERENCES}

[1] D. H. Armitage and S. J. Gardiner, Classical Potential Theory, Springer Monographs in Mathematics, Springer-Verlag London, Ltd., London, 2001.

[2] P. Collet, S. Martínez and J. San Martín, Asymptotic behavior of a Brownian motion on exterior domains, Probab. Theory Related Fields 116 (2000), 303-316.

[3] J. L. Doob, Classical Potential Theory and its Probabilistic Counterpart, SpringerVerlag, Berlin, 2001.

[4] J. Moser, A Harnack inequality for parabolic differential equations, Comm. Pure Appl. Math. 17 (1964), 101-134.

[5] R. G. Pinsky, Positive Harmonic Functions and Diffusion, Cambridge Studies in Advanced Mathematics 45, Cambridge University Press, Cambridge, 1995.

[6] S. C. Port and C. J. Stone, Brownian Motion and Classical Potential Theory, Academic Press, New York, 1978.

[7] N. Trudinger, Pointwise estimates and quasilinear parabolic equations, Comm. Pure Appl. Math. 21 (1968), 205-226. 
K. Wong

Department of Mathematics

Faculty of Science

Chulalongkorn University

Bangkok, 10330

Thailand

E-mail: kittipat.w@chula.ac.th

Received: 19.1.2005.

Revised: 19.7.2005. 